INRA Prod. Anim., 2017, 30 (1), 47-56

\section{La résistance génétique aux infections par les nématodes gastro-intestinaux chez les petits ruminants : un enjeu de durabilité pour les productions à l'herbe}

\author{
C. MORENO-ROMIEUX', G. SALLE' ${ }^{5}$, P. JACQUIET ${ }^{3}$, A. BLANCHARD , C. CHYLINSKI \\ J. CABARET ${ }^{5}$, D. FRANCOIS ${ }^{1}$, M. SACCAREAU ${ }^{1}, J .-M$. ASTRUC, \\ J.-C. BAMBOU ${ }^{2}, N . M^{2}$ ANDONNET ${ }^{2}$ \\ ${ }^{\prime}$ GenPhySE, Université de Toulouse, INRA, INPT, INP-ENVT, 31320, Castanet Tolosan, France \\ 2 INRA, Unité de Recherches Zootechniques, 97170, Petit-Bourg, France \\ ${ }^{3}$ INRA-ENVT, Interaction hôte-agent pathogène, 31076, Toulouse, France \\ 4 IDELE, Institut de l'élevage, 31326, Castanet Tolosan, France \\ ${ }^{5}$ INRA, Université de Tours, Infectiologie et santé publique, 37380, Nouzilly, France \\ Courriel : carole.moreno-romieux@inra.fr
}

Les infestations par les nématodes gastro-intestinaux sont source de pertes économiques importantes pour les élevages de petits ruminants conduits à l'herbe. La sélection d'animaux résistants aux nématodes gastro-intestinaux constitue un levier d'action prometteur à utiliser dans une gestion intégrée du parasitisme en élevage de petits ruminants.

Les « nématodes gastro-intestinaux » (notés NGI par la suite) ou strongles digestifs sont les principaux parasites internes des petits ruminants nourris à l'herbe (Mandonnet et al 2005, Davies et al 2006). Ces infestations engendrent des pertes économiques majeures, principalement dues aux pertes de production et aux coûts des traitements. Pendant longtemps, les traitements anthelminthiques ont été un moyen de lutte prédominant et efficace contre les NGI. Mais des résistances des NGI de petits ruminants à la plupart des molécules anthelminthiques sont observées à travers le monde (Kaplan et Vidyashankar 2012) et en France (Geurden et al 2014). De plus, certaines de ces molécules présentent une écotoxicité tout particulièrement pour la faune des sols (Lumaret et Errouissi 2002).

Dans ce contexte, la sélection génétique offre une opportunité durable d'améliorer la santé des animaux. On peut jouer sur deux leviers : la résilience ou la résistance. La capacité de résilience permet à l'hôte de continuer à produire malgré les infections parasitaires par les NGI mais cet hôte résilient reste une source de contamination pour ces congénères. La capacité de résistance permet à l'hôte de limiter voir d'empêcher l'installation et/ou la reproduction des NGI ; du coup l'hôte résistant permet de limiter la contamination de ces congénères. La sélection d'animaux génétiquement résistants semble la voie à privilégier. La gestion des infestations par les strongles gastrointestinaux doit désormais s'appuyer sur la combinaison raisonnée de plusieurs stratégies : la gestion du pâturage, les traitements anthelminthiques sélectifs et raisonnés, l'utilisation de fourrages bioactifs comme les plantes à tanins, la résistance/résilience de l'hôte aux parasites. La résistance génétique aux NGI s'inscrit dans cette démarche intégrée et y tient un rôle incontournable en complémentarité avec les autres stratégies (Jacquiet et al 2009). C'est une voie pour rééquilibrer de façon graduelle les relations hôte/parasite, au profit de l'hôte.

L'objectif de cette synthèse est de présenter les travaux menés sur la résistance génétique aux strongles gastro-intestinaux chez les ovins et caprins et de faire le point sur les stratégies de sélection d'animaux résistants dans une démarche de gestion intégrée de ces parasites.

\section{1 / Comment mesurer la résistance aux nématodes gastro-intestinaux ?}

Les NGI regroupent une dizaine d'espèces de vers localisés dans la caillette et les intestins des ovins et caprins. Les 3 espèces majeures observées et causant les pertes de production les plus importantes en régions tempérées sont : Teladorsagia circumcincta, Trichostrongylus colubriformis et Haemonchus contortus. En régions tropicales, l'espèce $T$. circumcincta est le plus souvent absente. Ces parasites ont tous un cycle de vie incluant une phase libre et une phase à l'intérieur du tube digestif de l'hôte. Les différentes étapes du cycle parasitaire s'accomplissent en environ un mois et sont décrites dans la figure 1 .

\section{1 / Mesure de la résistance des hôtes et protocole d'infestation}

La première étape pour la prise en compte de la résistance aux NGI en sélection consiste à définir le caractère que l'on veut améliorer, ainsi que les conditions de réalisation des mesures. La résistance, définie comme la capacité de l'hôte à réduire le nombre et/ou la fécondité des parasites qui le colonisent, est communément mesurée par des comptages d'œufs de NGI dans les fèces (OPG, œufs par gramme) lors d'une coproscopie (Kerboeuf et al 1997). Une variabilité individuelle importante des OPG est observée et ce caractère obéit à un déterminisme génétique modéré. Un autre critère souvent mesuré est l'hématocrite sanguin qui permet d'évaluer 
Figure 1. Cycle parasitaire d'un nématode gastro-intestinal.

Le cycle comprend quatre stades larvaires L1 à L4, puis des adultes qui éliminent des œufs dans les matières fécales. Ces œufs peuvent être comptés (OPG : œuf/g de fèces).

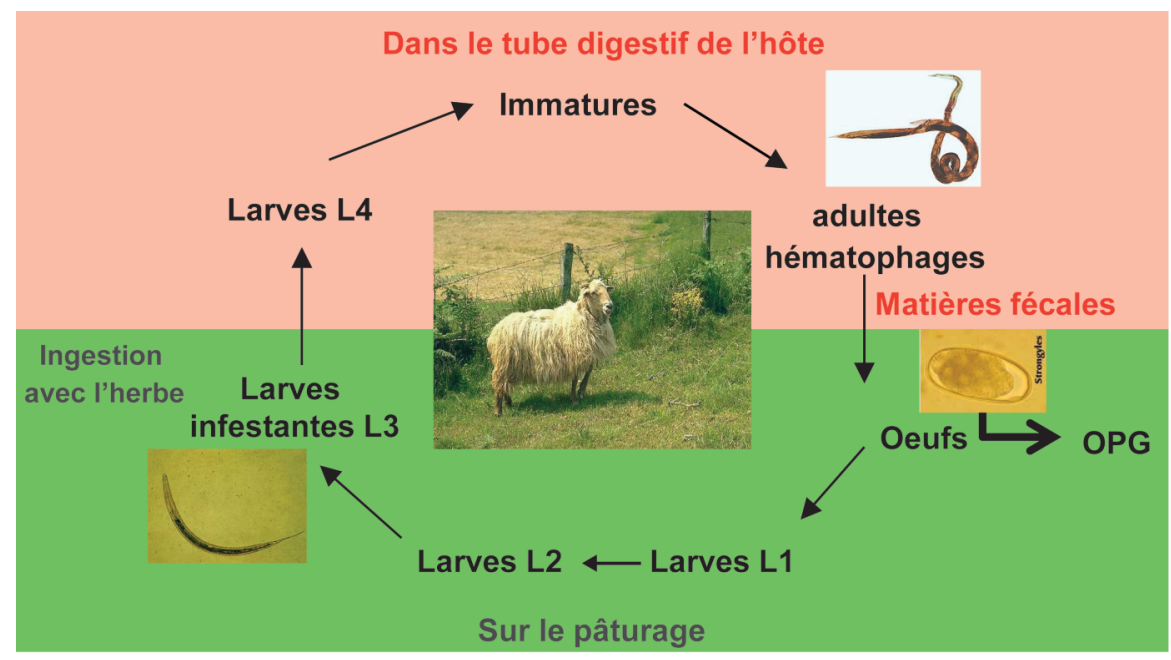

l'effet d'un ver hématophage comme Haemonchus contortus. Ce critère est une mesure de la résilience car il mesure la capacité de l'hôte à maintenir ses constantes biologiques et son niveau de production malgré l'infestation. Pour mesurer ces caractères, il semble plus simple de réaliser des prélèvements sur des individus au pâturage. Cependant, lors d'infestations naturelles, on ne mâ̂trise ni l'espèce de nématode ni le nombre de larves infestantes ingérées par l'hôte, ni la climatologie qui influe sur leur développement. L'alternative est de mesurer ces caractères (OPG et hématocrite par exemple) hors pâturage dans un protocole incluant deux infestations expérimentales successives avec une dose déterminée de larves infestantes d'un NGI (généralement Haemonchus contortus) (Gruner et al 2004a). Ce protocole est appliqué sur des animaux immunitairement naïfs de plus de trois mois n'ayant jamais pâturé et donc n'ayant jamais rencontré le parasite. La primo-infestation permet de mettre en place une réponse immunitaire innée qui mime la résistance des jeunes lors de leur première mise à l'herbe. La seconde infestation permet d'observer la résistance de l'hôte lors d'un second contact avec le parasite et ainsi l'efficacité de la réponse immune adaptative. Le nombre de larves infestantes ingérées par les animaux est choisi pour provoquer une réponse de l'hôte, sans pour autant causer un impact négatif sur la santé et la croissance des animaux.

Le protocole d'infestation expérimentale est désormais bien décrit. La dose de larves infestantes doit être optimale pour observer une variabilité de l'OPG dans les fèces et donc de la résistance des hôtes mais sans influer sur les caractères de production et de reproduction mesurés simultanément chez les béliers en station. Après plusieurs essais en situation expérimentale à l'INRA et Fédatest (races Romane et Blanc du Massif Central) et en stations de contrôle individuel des races Romane et Manech Tête Rousse, des doses d'environ 3500 et 5000 larves en première et deuxième infestation respectivement ont été choisies. Le protocole d'infestation expérimentale est efficace pour mettre en évidence la variabilité individuelle, mais sa mise en œuvre n'est pas simple (production et entretien des larves infestantes, infestation des animaux, prélèvement des fèces et prise de sang, comptage des OPG et mesure de l'hématocrite dans un laboratoire d'analyse) et nécessite une main d'œuvre spécialisée. Il est donc indispensable, pour des mesures à grande échelle de mettre en place une plateforme de phénotypage spécialisée, et la filière génétique ovine travaille en ce sens.

Chez les ovins en France métropolitaine, en raison de la lourdeur de réalisation des protocoles d'infestation expérimentale permettant de mesurer de manière standardisée la résistance aux nématodes gastro-intestinaux, il n'est pas envisageable à ce jour de réaliser ce phénotypage d'animaux en ferme. La population cible est donc constituée des mâles regroupés dans le même environnement en station, c'est à dire des mâles importants au plan génétique, qui vont diffuser dans la population raciale.

Par ailleurs, pour les races ovines d'herbage dont les béliers regroupés en station sont conduits au pâturage, la mise en œuvre du phénotypage est délicate en France métropolitaine : l'infestation naturelle ou les essais de contamination des prairies se sont avérés rarement concluants car très dépendants des conditions météorologiques (peu de contamination sur les pâtures lors de périodes très sèches et au contraire nécessité de traiter les animaux lors de périodes très humides). Il faut noter que dans des régions plus humides et chaudes (par exemple en Guadeloupe, en Uruguay ou dans certaines zones d'Australie ou de Nouvelle Zélande) où la pression parasitaire naturelle est constante et forte, des mesures en infestation naturelle sont réalisées à grande échelle pour la sélection d'animaux résistants (Karlsson et Greeff 2006, Ciappesoni et al 2010, Gunia et al 2013a, Pickering et al 2015).

Le frein principal actuel à la diffusion à grande échelle de la mesure de la résistance aux NGI en France est la lourdeur et le coût d'obtention du phénotype du nombre d'œufs de nématodes dans les fèces. Un protocole d'infestation expérimentale avec mesure du nombre d'œufs dans les fèces et de l'hématocrite a un coût d'environ $50 €$ par candidat testé en France. Il s'agit d'un investissement non négligeable pour les structures de sélection, même si le bénéfice attendu, qui reste à chiffrer, d'une évolution génétique à moyen/long terme à l'échelle de la population raciale (diminution des coûts de traitements, diminution de la résistance des vers aux anthelminthiques, moindre réforme et moindre perte de production liées au parasitisme par les NGI) est sans doute positif.

\section{2 / D'autres mesures de la résistance dans l'avenir}

\section{a) Méthodes d'automatisation des comptages d'oufs}

Pour pallier la lourdeur des protocoles de phénotypage, liée à des modes opératoires peu ou pas automatisés, différents axes d'étude sont actuellement envisagés. À ce jour, l'automatisation de la coproscopie par analyse d'image reste expérimentale (Mes et al 2007). Cependant, une méthode basée sur le marquage des œufs de strongles à l'aide d'une lectine fluorescente est désormais disponible en Australie et semble faciliter substantiellement la coproscopie tout en permettant de distinguer les espèces présentes (Hillrichs et al 2012).

Une autre façon de compter le nombre d'œufs dans les fèces a récemment été proposée : c'est la coproscopie moléculaire (Roeber et al 2012, Demeler et al 2013). Cette technique permet de quantifier l'excrétion d'œufs sur la base de 1'ADN des NGI par PCR (Polymerase Chain Reaction) en temps réel, au lieu d'un comptage au microscope et d'une identification des espèces de NGI présentes dans les fèces. Les premiers résultats sont encourageants mais présentent des résultats contradictoires avec la 
méthode de référence de comptage d'œufs au microscope dans environ $5 \%$ des cas (peut-être du fait de la présence d'inhibiteurs de la PCR dans les matières fécales). Cette approche moléculaire permettrait d'automatiser les mesures et pourrait être réalisée sur des échantillons de fèces congelés, ce qui faciliterait la réalisation des mesures dans le temps.

b) Autres méthodes : antigène et métabolome

D'autres pistes de recherche sont actuellement explorées pour identifier des méthodes à la fois moins coûteuses et plus simples à mettre en œuvre, qui maintiendraient le niveau de sensibilité et de spécificité requis pour le phénotypage. En étudiant la réponse immunitaire contre les nématodes trichostrongles (T. colubriformis et $H$. contortus), des travaux ont mis en évidence un antigène spécifique des larves des nématodes trichostrongles, appelé CarLA, reconnu par le système immunitaire (Harrison et al 2008). Cette réaction entraîne notamment la production d'IgA anti-CarLA, dont la concentration peut facilement être quantifiée par un test salivaire (Shaw et al 2012). Cependant la corrélation génétique avec les OPG n'est que de 0,50 , ce qui s'explique par le fait que ce critère ne semble être affecté que lors de l'installation des larves et pas en présence des vers adultes. D'autres travaux se focalisent sur la recherche de mesures de la résistance plus génériques et moins coûteuses : les biomarqueurs. L'idée est, par exemple, d'étudier l'ensemble des métabolites d'un individu, et donc les variations biochimiques associées à l'infestation ou non par des nématodes. À ce jour, seul un nombre limité de travaux se sont intéressés aux perturbations des profils métabolomiques associés aux infestations par des nématodes et aucun n'a été réalisé chez les petits ruminants (Wang et al 2010). Par exemple, des marqueurs urinaires d'infestation par des nématodes ont ainsi pu être mis en évidence chez l'Homme et le Hamster. Cette approche est actuellement à l'étude dans le cadre du projet ICSA GEMANEMA (coordonné par G Sallé) pour identifier des biomarqueurs d'infestation chez les ovins.

\section{2 / Génétique de la résistance aux nématodes gastro-intes- tinaux (NGI)}

\section{1 / La résistance aux NGI est- elle sous contrôle génétique ?}

Les résultats de la recherche démontrent la possibilité de sélectionner efficacement sur la résistance aux NGI chez les ovins et les caprins en mesurant les OPG (tableau 1). En effet, 1'héritabilité de ce caractère est modérée $(0,2-0,4)$ avec une composante génétique maternelle (anticorps de la mère) qui disparait avec la maturation du système immunitaire des chevreaux (après l'âge de 6 mois) et agneaux (après l'âge de 3 mois) (Bishop et Morris, 2007, de la Chevrotiere et al 2012, Assenza et al 2014). La résistance est fortement corrélée génétiquement entre infestations artificielle et naturelle (la corrélation est de 0,87 chez les ovins et de 0,53 chez les caprins) et entre les infestations par différents parasites (la corrélation est de 1 entre les deux parasites majeurs des ovins : T. colubriformis et $H$. contortus) (Gruner et al 2004a et b). Ce dernier point est majeur : la résistance à une espèce de NGI est compatible avec la résistance à une autre espèce de NGI. Les corrélations entre stades de production sont élevées et favorables (la corrélation est supérieure à 0,7 en caprin). Ainsi, une sélection sur les chevrettes en engraissement permettra de limiter l'intensité et la persistance du pic d'excrétion des futures chèvres adultes autour du part. Les corrélations génétiques avec la fertilité, la valeur laitière sont proches de zéro (Gunia et al 2011). En revanche, les corrélations génétiques entre résistance et poids vif varient de valeurs négatives favorables (lorsque les animaux sont infestés ou pas) à des valeurs légèrement positives défavorables (lorsque les animaux ne sont pas infestés) (Gunia et al 2011, Assenza et al 2014). Doeschl-Wilson et al (2008) ont montré par modélisation que ces corrélations génétiques variables sont certainement dues à des stratégies d'allocations de ressources alimentaires variables (selon la race étudiée et/ou les ressour- ces alimentaires disponibles). Par ailleurs, à ce jour, aucune étude n'a permis de démontrer que la sélection pour la résistance au NGI pourrait avoir un effet défavorable sur la résistance à d'autres pathologies (Hunt et al 2013).

L'héritabilité des OPG, estimée en race laitière Manech Tête Rousse, (sur des béliers de 2 à 3 ans en centre d'élevage) est de l'ordre de ce que l'on obtient classiquement en deuxième infestation $(0,34)$ mais cette héritabilité est beaucoup plus faible en 1ère infestation $(0,11)$ (Astruc et al 2016). Les corrélations génétiques avec les caractères laitiers sont non significatives sauf pour le taux butyreux qui a une corrélation légèrement défavorable avec la resisitance aux NGI. Étant donné que la mesure d'OPG en deuxième infestation a une héritabilité modérée et qu'elle permet d'apprécier la résistance à des infestations successives, on pourrait se limiter à la mesure d'OPG à cette dernière infestation. Cette simplification permettrait de diviser par deux le nombre de mesures d'OPG et donc le coût du phénotypage. Ces résultats sont à confirmer dans d'autres races et notamment sur de jeunes béliers en station de contrôle individuel dans des races allaitantes.

Le protocole d'infestation expérimentale fournit également des mesures de la résilience des béliers, au travers de l'hématocrite. Ces mesures ont des héritabilités faibles à modérées $(0,10$ et 0,25 en races Manech Tête Rousse et Romane, respectivement) et sont modérément négativement corrélées aux OPG (- 0,40 en Romane) (Sallé et al 2012, Assenza et al 2014). Un index combiné global pourrait être pris en compte en considérant un critère rassemblant résistance (OPG) et résilience (hématocrite).

Les mesures indirectes concernent essentiellement l'effet du parasitisme par les NGI sur la production (gains de poids, condition corporelle) ou la pathologie (indice d'anémie-hématocrite qui concerne surtout Haemonchus contortus ou bien un indice de diarrhée). Ces mesures ne sont pas toujours bien reliées avec l'infestation (Chylinski et al 2015).

Tableau 1. Principaux paramètres génétiques de la résistance génétique aux nématodes gastro-intestinaux mesurée par les œufs de nématodes gastro-intestinaux dans les fèces les ovins (O) ou des caprins (C).

\begin{tabular}{|l|c|c|c|c|}
\hline & Héritabilité & $\begin{array}{c}\text { Corrélation entre } \\
\text { infestations par des } \\
\text { parasites différents }\end{array}$ & $\begin{array}{c}\text { Corrélation entre } \\
\text { infestations naturelle } \\
\text { et expérimentale }\end{array}$ & $\begin{array}{c}\text { Corrélation entre } \\
\text { résistance du jeune } \\
\text { et de l'adulte }\end{array}$ \\
\hline $\begin{array}{l}\text { Nombre d'œuf } \\
\text { dans les fèces } \\
\text { (OPG) }\end{array}$ & $0,2-0,4(\mathrm{C}$ et $\mathrm{O})$ & $1(\mathrm{O})$ & $0,53(\mathrm{C}) 0,87(\mathrm{O})$ & $0,7(\mathrm{C})$ \\
\hline & $\begin{array}{c}\text { de la Chevrotière et } \\
\text { al }(2011)\end{array}$ & Gruner et al (2004a) & $\begin{array}{c}\text { Bambou et al }(2010) \\
\text { Gruner et al }(2004 \mathrm{~b})\end{array}$ & $\begin{array}{c}\text { Mandonnet et al } \\
(2006)\end{array}$ \\
\hline
\end{tabular}


En conclusion, résistance et résilience aux NGI présentent une grande variabilité génétique qui se traduit par des traits d'histoire de vie différents, tels qu'une plus ou moins grande capacité à envahir l'hôte/à être fertile (IgA, OPG) ou à produire des effets pathologiques (anémie évaluée à travers l'hématocrite...). L'ensemble de ces paramètres est sélectionnable. Cependant, la sélection pour la résistance (via la mesure d'OPG) est la stratégie la plus efficace car elle permet de sélectionner à la fois des animaux moins parasités et potentiellement moins malades mais aussi moins contaminants pour les pâtures et donc pour leurs congénères.

\section{2 / Quels sont les mécanismes biologiques de la résistance géné- tique au parasitisme par les NGI ?}

La résistance vis-à-vis des infestations par les NGI chez les ruminants se traduit entre autres par une diminution du nombre d'œufs de nématodes excrétés par l'animal. Cette mesure reste actuellement la plus utilisée pour mesurer les niveaux d'infestation chez des animaux vivants, et par conséquent la résistance de l'hôte. La réponse immunitaire contre les NGI, en relation avec une baisse de l'OPG est bien étudiée chez les ovins (Miller et Horohov 2006). Il a été montré que la résistance, à la fois contre l'installation des larves infestantes et contre les nématodes adultes, est liée à la mise en place d'une réponse immunitaire adaptative. De nombreuses zones du génome (« quantitative trait loci » : QTL) associées à la résistance ont été identifiées dans plus de 20 régions chromosomiques dans différentes races ovines (Bishop et Morris 2007, Kemper et al 2011, Sallé et al 2012, Riggio et al 2013, McRae et al 2014). L'implication de gènes candidats situés dans des zones du génome identifiées tels que l'interféron gamma et le complexe majeur d'histocompatibilité a été suggérée probablement pour leur rôle connu dans la réponse immunitaire. L'étude fine de la région QTL du chromosome 12 réalisée par Sallé et al (2014) a permis de démontrer que les animaux porteurs des allèles de résistance dans cette région avaient une réponse immunitaire Th-2 quantitativement plus importante, et une fécondité des vers réduite. La réponse de type Th- 2 est adaptative et se caractérise par la production de diverses interleukines ; elle est particulièrement sollicitée lors d'infestation par les helminthes, dont les nématodes gastro-intestinaux. De manière générale, aucun gène à effet majeur n'a pu être identifié dans toutes ces études. À partir de lignées ovines sélectionnées de manière divergente pour la résistance aux NGI à partir de mesures OPG, une région a été identifiée sur le chromosome
19, à proximité du gène HRH1, associe à une réponse immune de type Th-1 (McRae et al 2014). La réponse Th-1 correspond à une réponse adaptative en cas de réinfection; elle se caractérise par la production d'interféron gamma et abouti à une réponse cellulaire particulièrement dirigée sur les pathogènes intra-cellulaires. Il est donc fort probable que l'héritabilité de la résistance aux NGI soit due à plusieurs gènes, chacun ayant un effet limité. De plus, une analyse conjointe menée dans trois populations européennes d'ovins issues d'Écosse, de France et d'Espagne, a permis de mettre en évidence des régions chromosomiques communes entre plusieurs races sur les chromosomes 4, 12, 14, 19 et 20 (Riggio et al 2014).

Chez les caprins, une dizaine de QTL associés à la résistance et à la résilience ainsi qu'à la réponse humorale anti- $H$. contortus ont été identifiés dans la seule étude menée à ce jour (de la Chevrotière et al 2012).

Malgré le nombre croissant d'études comparant des animaux résistants et sensibles entre races ou au sein d'une même race, il n'existe pas de consensus clair sur les mécanismes clés à l'origine de la résistance génétique. Des travaux récents montrent qu'étudier la cinétique de la réponse protectrice pourrait être plus pertinent que de se focaliser uniquement sur son intensité (Robinson et al 2010a et b). Des études longitudinales couplant l'utilisation de modèles animaux originaux (lignées divergentes) et des approches « omiques » (transcriptomiques, métabolomiques) apparaissent comme une perspective très prometteuse.

\section{3 / Comment gérer le risque de contournement de la résistance par le parasite ?}

La sélection d'hôtes résistants aux NGI est une perspective intéressante pour l'élevage. Mais le parasite dispose d'une multitude de gènes lui permettant de s'adapter à son milieu (dont l'hôte fait partie). Des études ont montré que la diversité génétique chez $H$. contortus est extrêmement élevée, même à l'échelle individuelle (Otsen et al 2001). Les travaux en cours visent donc à comprendre les régulations transcriptomiques qui interviennent dans différents contextes de pression de sélection, dont la résistance génétique de l'hôte. De cette façon, il sera envisageable d'élaborer des modèles prédictifs pour déterminer les pratiques qui risquent de conduire à une évolution vers des populations de nématodes plus virulentes.

Un des moyens de prendre en compte l'aspect évolutif du couple hôte-parasite est la mesure de la fitness (nombre de larves infestantes produites par l'hôte/ nombre de larves infestantes ingérées). Le NGI $H$. contortus, quel que soit l'isolat (sensible, résistant ou multi-résistant aux anthelminthiques), possède une grande capacité d'adaptation lui permettant d'ajuster ses traits de vie pour maintenir une fitness lorsqu'il se développe sur fond génétique hôte sensible ou résistant (Chylinski 2014). La fitness des NGI est donc la mesure adéquate pour évaluer l'évolution au cours du temps des NGI face à des petits ruminants résistants. Schmidt (1998) a mesuré l'évolution de $T$. circumcincta et montré que les nématodes issus d'une sélection chez des ovins résistants avaient une meilleure fitness que ceux qui étaient issus d'ovins sensibles. Cette adaptation n'a pas été remarquée par Saulai et al (2001) chez H. contortus. L'évolution de la fitness est donc variable et des études complémentaires s'avèrent nécessaires.

Le triangle nématode-hôte-environnement doit être considéré dans le cadre d'approches d'écologie évolutive pour déterminer le risque de contournement associé à chaque pratique mise en place pour contraindre le parasite. D'une manière générale, la durabilité des stratégies de contrôle des NGI repose sur la combinaison de différentes méthodes de manière à éviter la pression de sélection sur un facteur et diminuer les risques de contournement : l'utilisation des résistances de l'hôte, la gestion du pâturage, la complémentation alimentaire, la chronologie des traitements anthelminthiques, par exemple. La combinaison des dispositifs disponibles doit être étudiée pour évaluer l'impact sur le parasite des différentes méthodes, seules, alternées ou simultanées. L'objectif est de définir la stratégie optimale qui s'appuie sur un concept de contraintes diversifiées et raisonnées limitant ainsi la pression sur le parasite qui le conduit à évoluer et devenir plus virulent. Actuellement nous n'avons ni recul ni données sur ces aspects d'association de stratégies. En revanche, l'emploi exclusif d'une stratégie risque de conduire à un contournement de la part du parasite. Cela a été démontré très clairement chez les nématodes de plantes sur lesquels il est plus facile de suivre les générations et leur évolution (McDonald et al 2002, DjianCaporalino et al 2014).

Le risque de contournement suite à une sélection génétique reste limité en pratique chez les petits ruminants. Premièrement parce que la pression de sélection sur les populations de petits ruminants sera faible (l'index prend aussi en compte d'autres objectifs de sélection comme les caractères de production) mais aussi parce que cette résistance est déterminée par plusieurs gènes (poly- 
génique) et donc repose sur plusieurs mécanismes. D'autre part, nous ne sommes pas intéressés par une pression de sélection trop forte puisqu'on recherche un équilibre entre l'hôte et le pathogène et non pas une éradication. Il est toutefois nécessaire de contrôler l'évolution des aptitudes des populations de vers afin de vérifier, voire d'anticiper ce phénomène de contournement.

\section{3 / Comment utiliser la résis- tance génétique au parasiti- sme par les nématodes gastro- intestinaux en élevage?}

Nous avons vu dans le paragraphe précédent que la résistance au parasitisme gastro-intestinal chez les petits ruminants était sous contrôle génétique et faiblement corrélée génétiquement aux autres caractères en sélection. C'est donc une situation favorable pour mettre en place une sélection sur ce caractère.

\section{1 / Démonstration de l'efficacité de la sélection}

Plusieurs études en France et à l'étranger ont cherché à évaluer l'efficacité d'une sélection sur la résistance aux NGI en étudiant des animaux génétiquement extrêmes. La création d'animaux génétiquement divergents (tableau 2) est une démonstration expérimentale indiscutable puisqu'elle mime une sélection accélérée de ce qui pourrait être mis en place dans les schémas de sélection.

Chez les ovins allaitants, des lignées divergentes basées sur la sélection des OPG issus de deux infestations successives sur 280 agneaux Romanes de six mois ont été créées au domaine expérimental INRA de Bourges (Moreno et al 2016). Trois pourcents des pères extrêmes et $30 \%$ des mères extrêmes ont été utilisés pour faire la première génération de sélection. Les animaux issus de la lignée résistante excrètent en moyenne six fois moins d'œufs que ceux issus de la lignée sensible. La divergence en première génération est de plus de trois écarts types génétiques. Ces résultats démontrent l'efficacité potentielle d'une sélection génétique en race Romane. Dans le tableau 1 sont présentées les différentes lignées divergentes sur la résistance aux NGI à travers le monde. On peut voir qu'avec une pression de sélection forte et un phénotype plus précis (OPG après infestations expérimentales), les lignées françaises ont divergé quasiment autant en une génération que les lignées Néo-Zélandaises en trois générations et légèrement moins que les lignées Australiennes en 10 générations. Ces lignées sont aussi un formidable support pour l'étude de l'impact de la sélection à long terme sur d'autres caractères d'intérêt tel que des caractères d'adaptation (quel serait l'impact d'une restriction alimentaire ou d'un autre stress sur le niveau de résistance des animaux ?) ou sur les traits de vie du ver (quel est le(s) trait(s) de vie affecté(s) par la résistance génétique : l'établissement des larves infestantes, le développement de la larve au ver adulte ou bien encore la fécondité des vers femelles; l'adaptation du ver aux hôtes résistants est-elle possible?).

Chez des caprins Créole, l'efficacité d'une indexation sur le caractère de résistance a été testée sur l'élevage de l'unité expérimentale INRA de Guadeloupe par la création de lignées divergentes. Il ressort de cette étude qu'une différence d'index relativement modeste sur l'excrétion d'œufs à l'âge de 11 mois (0,53 écart-type génétique en moyenne) entre deux troupeaux de chèvres mères s'est traduite par une diminution de $32 \%$ de l'excrétion des OPG autour du part pour les mères sélectionnées (Blaes et al 2010).
Dans une race ovine laitière des Pyrénées, la Manech Tête Rousse, une sélection chez des béliers d'insémination artificielle a été réalisée et a permis d'identifier des individus extrêmes (50\% plus résistants versus $50 \%$ plus sensibles). Les filles de ces béliers ont été suivies dans sept élevages commerciaux du département des Pyrénées Atlantiques sur toute une saison de pâturage. Des mesures d'OPG réalisées chez les filles en conditions naturelles d'infestation ont permis de montrer que les brebis issues des béliers résistants excrétaient plus deux fois moins d'œufs dans les fèces que les brebis issues des béliers sensibles (figure 2 ; Jacquiet et al 2015). Ces résultats permettent de valider l'intérêt et l'efficacité de la sélection sur les béliers en station pour améliorer la résistance des brebis dans les élevages.

Chez les ovins ou les caprins, la sélection opérée sur le critère d'OPG est donc réellement efficace à différents stades de production. De plus, un gain de production a pu être observé en caprin Créole. En effet, le poids de chevreaux sevrés est $16 \%$ plus élevé lorsqu'ils sont élevés par une mère résistante (Mandonnet et al 2014). En conclusion, l'animal résistant présente deux avantages : premièrement, il est moins infesté et donc maintient mieux son niveau de production ; deuxièmement il excrète moins d'œufs dans son environnement et donc limite la contamination du reste du troupeau.

\section{2 / L'intégration de la résistance aux nématodes gastro-intestinaux dans les schémas de sélection}

La seconde étape consiste à combiner ce caractère de résistance avec les autres caractères d'intérêt pour les éleveurs, dans un objectif d'optimisation du profit de l'atelier d'élevage.

Tableau 2. Les principales lignées divergentes pour la résistance aux Nématodes Gastro-Intestinaux (NGI) à travers le monde.

\begin{tabular}{|c|c|c|c|c|c|c|c|c|}
\hline Pays & $\begin{array}{l}\text { Espèce I } \\
\text { race }\end{array}$ & Infestations & Parasites & $\begin{array}{l}\text { Nombre } \\
\text { d'années }\end{array}$ & $\begin{array}{l}\text { Critères } \\
\text { de } \\
\text { sélection }\end{array}$ & $\begin{array}{l}\text { Pression de sélection } \\
\text { à chaque génération }\end{array}$ & $\begin{array}{l}\text { Divergence } \\
\text { (en SigA*) }\end{array}$ & Références \\
\hline France & $\begin{array}{c}\text { Ovin / } \\
\text { Romane }\end{array}$ & artificielle & H. contortus & 2 & OPG** & $\begin{array}{l}3 \% \text { des males } \mathrm{R} / \mathrm{S}^{* * *} \\
30 \% \text { des femelles } \mathrm{R} / \mathrm{S}\end{array}$ & 3 & $\begin{array}{l}\text { Moreno } \\
\text { et al (2016) }\end{array}$ \\
\hline Australie & $\begin{array}{l}\text { Ovin / } \\
\text { Merinos }\end{array}$ & artificielle & $T$ colubriformis & 20 & $\mathrm{OPG}^{* *}$ & $\begin{array}{l}12 \% \text { des males R/S } \\
50 \% \text { des femelles R/S }\end{array}$ & 4,7 & $\begin{array}{c}\text { Woolaston } \\
\text { et Windon } \\
(2001) \\
\end{array}$ \\
\hline $\begin{array}{l}\text { Nouvelle- } \\
\text { Zélande }\end{array}$ & $\begin{array}{l}\text { Ovin / } \\
\text { Romney }\end{array}$ & naturelle & $\begin{array}{l}\text { Multi espèces } \\
\text { de NGI }\end{array}$ & 7 & OPG ** & $\begin{array}{c}25 \% \text { des males } \mathrm{R} / \mathrm{S} \\
\text { Pas de sélection femelle }\end{array}$ & 2,9 & $\begin{array}{c}\text { Morris } \\
\text { et al (1997) }\end{array}$ \\
\hline France & $\begin{array}{l}\text { Caprin / } \\
\text { Chèvre } \\
\text { Créole }\end{array}$ & artificielle & H.contortus & 4 & OPG ** & $\begin{array}{l}12 \% \text { des mâles R/S } \\
50 \% \text { des femelles R/S }\end{array}$ & 2,6 & $\begin{array}{l}\text { Bambou } \\
\text { et al (2013) }\end{array}$ \\
\hline
\end{tabular}

${ }^{*}$ SigA : écart type génétique ; ${ }^{*} \mathrm{OPG}:$ Nombre d'œufs de nématodes gastro-intestinaux dans les fèces ; ${ }^{* *} \mathrm{R}:$ résistant ; $S$ : sensible. 
Figure 2. Illustration de l'impact en fermes de la sélection sur la résistance aux NGI chez les béliers de race Manech Tête Rousse en station de testage.

${ }^{* * *}$ : Signifie que cette différence est significative au seuil de $0,1 . \quad$;opg : Nombre d'oeufs de nématodes gastro-intestinaux dans les fèces.

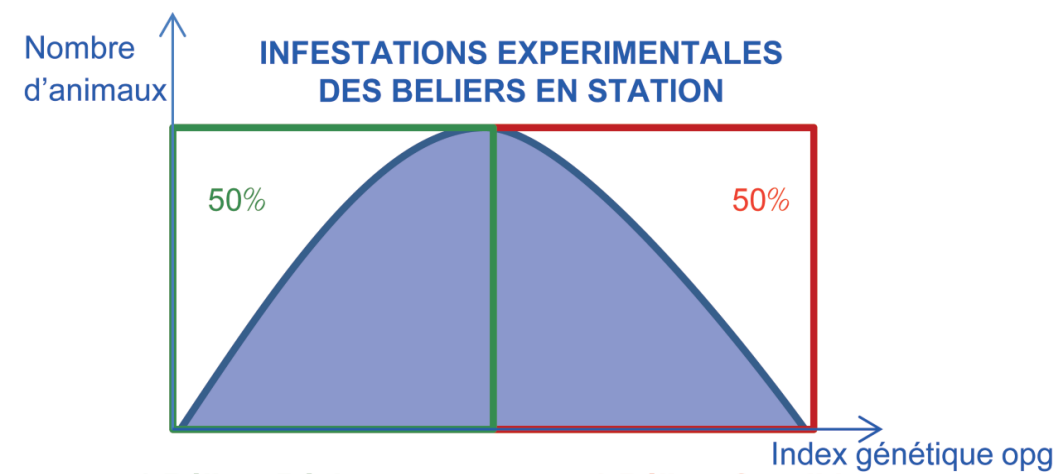

50 Béliers Résistants

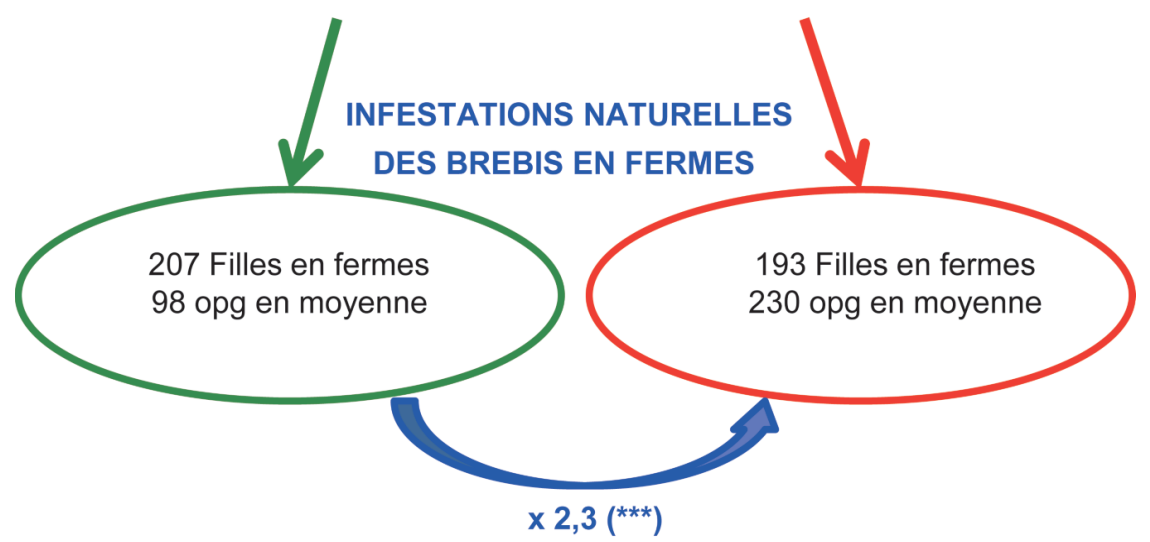

Chez les caprins, une démarche de recherche participative avec les éleveurs de la coopérative Cabricoop, a permis de concevoir un schéma de sélection adapté à l'élevage des caprins Créoles au pâturage en Guadeloupe. À partir du modèle bioéconomique déterministe établi à cette occasion (Gunia et al 2013a), un objectif de sélection a été construit intégrant la résistance aux NGI (OPG et hématocrite) et des caractères de production (poids vif rendement carcasse à l'âge de 11 mois, de reproduction, fertilité) (Gunia et al 2013b). Les poids économiques optimums ont été estimés. L'inclusion de la résistance aux NGI induit une perte de progrès génétique modeste sur les autres caractères de production en sélection (- $2 \%$ et $-6 \%$ de progrès génétique en moins pour le poids vif et le rendement carcasse respectivement). Cependant les caprins Créole évoluant dans un milieu fortement parasité, l'utilisation d'animaux plus résistants engendre un gain de productivité pour l'éleveur en limitant les pertes de production liées aux NGI. La sélection ainsi proposée aux éleveurs rend compatible une production accrue avec une amélioration significative de la résistance à long terme.

Par ailleurs, dans plusieurs races ovines de métropole (Manech Tête Rousse et Romane), des travaux sont en cours pour nécessaires à la prise en compte en sélection de la résistance aux NGI. En Manech Tête Rousse, des évaluations génomiques vont être réalisées puisque les béliers évalués sur la résistance aux NGI sont aussi génotypés avec des puces portant 54000 marqueurs SNP («Single nucleotide polymorphism »). Les indicateurs génétiques pourront être valorisés, soit $i$ ) de manière ponctuelle, en utilisant des mâles résistants (d'Insémination Artificielle de préférence) chez des éleveurs où la résistance aux anthelminthiques est avérée, soit $i i$ ) pour réaliser une sélection, via une pression de sélection sur un critère global incluant la résistance aux NGI.

L'Uruguay, l'Australie et la Nouvelle Zélande bénéficient d'un climat assez chaud et humide dans une partie de leurs territoires, propice aux infestations par les NGI. Ces conditions ont permis de tester une sélection sur la résistance génétique aux NGI basée sur des lignées (Morris et al 1997, Woolaston et Windon 2001) et de mettre en place une sélection réalisée à partir de coproscopies répétées d'animaux au pâturage en infestations naturelles (Ciappesoni et al 2010, Morris et al 2010).

\section{3 / Comment optimiser l'utilisa- tion de béliers résistants dans les troupeaux?}

affecter une pondération économique à la résistance aux NGI. Des approches sont possibles pour donner une valeur économique à un gain de résistance aux NGI selon différentes contraintes acceptables par l'éleveur (par exemple le nombre de traitements anthelminthiques visés). Néanmoins, au-delà des seules considérations économiques, il faudra ne pas négliger que la sélection pour la résistance aux NGI est une alternative vertueuse à la lutte chimique contre les NGI, s'inscrivant parfaitement dans le concept de l'agroécologie, avec sa double performance économique et environnementale. L'objectif de réduction des résidus d'anthelminthiques de synthèse dans les prairies et de préservation de la microfaune du sol doivent être intégrés.

Aujourd'hui, la sélection pour la résistance aux NGI n'est pas effective en routine en France, même si quelques races (Manech Tête Rousse et Romane) disposent de mesures non systématiques. La genèse en cours d'une plate-forme de phénotypage qui devrait s'appuyer sur l'école vétérinaire de Toulouse, pionnière en la matière, est une étape incontournable pour la mise en place d'un transfert de connaissances et de technologies aux éleveurs et organismes de sélection. La mise en place d'évaluation génétique va fournir aux organismes de sélection et aux entreprises de sélection les outils
La situation optimale et durable pour un élevage n'est pas la constitution d'un troupeau complètement indemne de parasites que ce soit à l'aide d'une lutte chimique ou par l'utilisation d'animaux résistants.

L'objectif est d'utiliser la résistance afin d'aboutir à un équilibre qui permette aux hôtes de maintenir leur production tout en soumettant les parasites à une pression de sélection raisonnable (en limitant l'installation et la multiplication des parasites). L'objectif ultime pour l'éleveur est que le troupeau contrôle suffisamment le parasite pour limiter la pression infectieuse et ainsi maintenir une bonne production sans traitement ou avec un nombre limité de traitements. Pour cela l'éleveur peut jouer sur plusieurs leviers, parmi lesquels ont été considérés les traitements anthelminthiques, la nutrition (quantité et qualité), la surface pâturée et la génétique de son troupeau.

À partir d'un modèle existant pour l'agneau en croissance (Laurenson et al 2012), un modèle «épidémio-génétique » a été développé pour prendre en compte ces leviers et ainsi modéliser l'évolution de l'infection au sein d'un troupeau constitué d'agneaux et de brebis en production (laitières ou allaitantes) (Sac- 
careau et al 2016). Ce modèle peut être paramétré de manière à prendre en compte les conditions d'élevage (taille du troupeau, qualité nutritive de l'aliment, taille de pâture, plein air intégral/ partiel), les caractéristiques des animaux (résistance génétique, ovins laitiers/ allaitants) et du nématode (isolat de vers, statut de résistance aux anthelminthiques, fréquence des traitements chimiques). L'ensemble de ces paramètres permet d'ajuster le modèle à des données réelles. Le modèle peut ensuite être utilisé pour tester in silico l'impact à plus ou moins long terme de différentes combinaisons de stratégies de lutte : pression de sélection sur les béliers génétiquement résistants, nombre des traitements chimiques de synthèse, nutrition, gestion des pâtures. L'évaluation expérimentale de ces stratégies et des combinaisons de stratégies seraient impossibles car extrêmement couteuses et longues à mettre en œuvre. Le modèle permet ainsi d'orienter le choix des stratégies à mettre en œuvre en fonction de l'objectif à atteindre pour l'éleveur. Une étude va être menée dans les Pyrénées Atlantiques dans la race Manech Tête Rousse dans les années à venir pour tester l'impact de la sélection génétique sur les conduites d'élevage (diminution de la fréquence des traitements par exemple).

\section{4 / Quelles stratégies de gestion intégrée?}

À partir des résultats présentés ici, la sélection pour la résistance aux NGI paraît une alternative possible au seul emploi des anthelminthiques. Cependant, elle ne sera pleinement efficace qu'en complémentarité avec d'autres pratiques d'élevage (Hoste et al 2010). Les travaux sur la génétique sont réfléchis dans une perspective agro-écologique de gestion de l'équilibre entre l'animal et son système d'élevage.

Ainsi, depuis quelques années, la stratégie de maîtrise des parasites NGI a évolué d'une logique d'élimination des parasites vers une logique de manipulation des équilibres hôtes-parasites dans les systèmes pâturés (Mahieu et al 2009). La gestion des NGI en élevage à l'herbe doit désormais reposer sur un panel d'outils combinant trois stratégies (figure 3). La première est une stratégie immédiate de réduction de la probabilité de rencontre entre l'hôte et le parasite, grâce à la gestion du pâturage (rotation, mixité des espèces ou stades physiologiques, fauche) (photo 1). Le risque parasitaire est alors dilué pour les animaux les plus sensibles. La deuxième stratégie, à moyen terme, vise à prolonger l'efficacité des molécules de synthèse en pratiquant des traitements sélectifs et en valorisant les effets anthelminthiques des métabolites secondaires présents dans certaines res-

Figure 3. Représentation des différents leviers d'action pour un contrôle intégré des nématodes gastro-intestinaux (NGI) (Source : N Mandonnet, INRA).

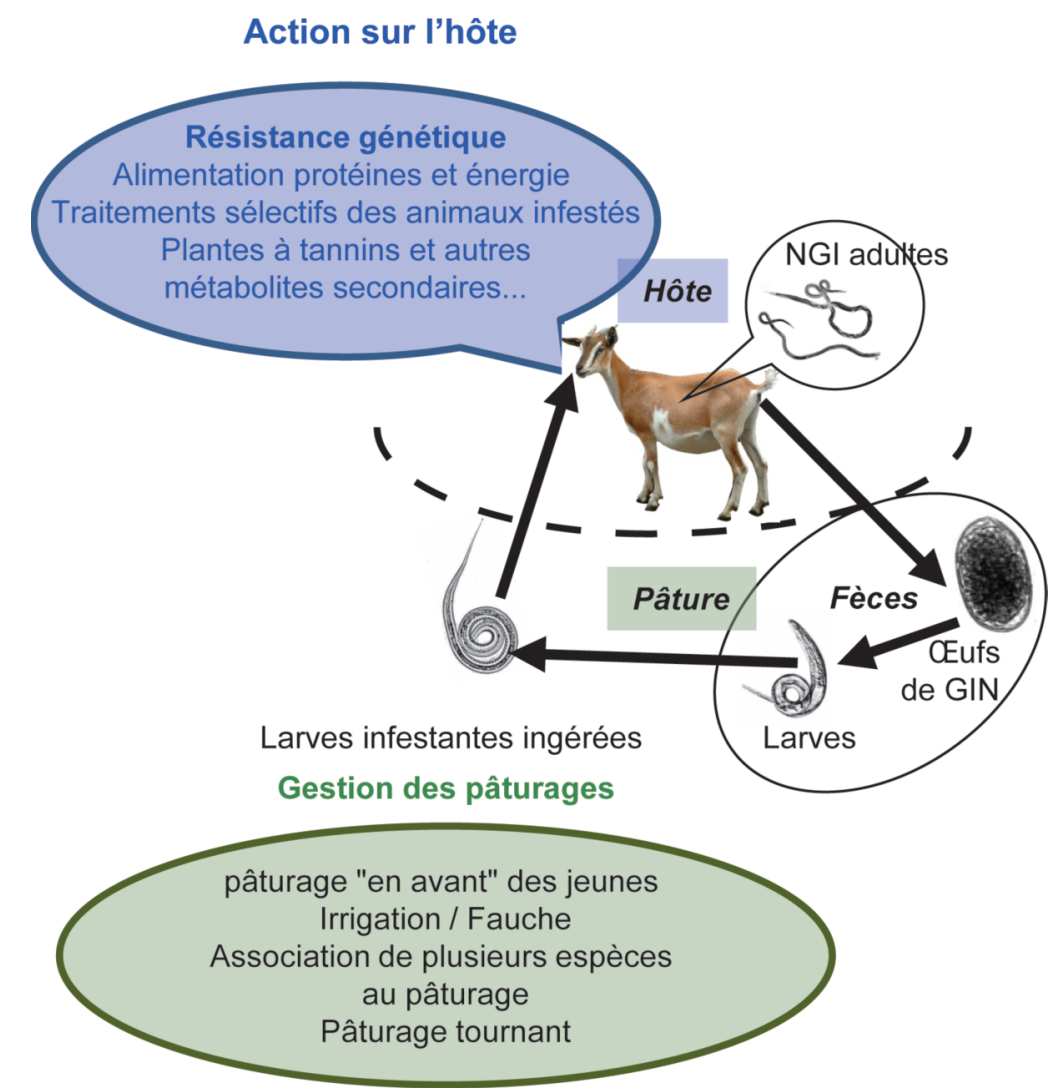

Photo 1. Pâturage mixte caprin-bovins pour réduire la contamination parasitaire chez les deux espèces (Photo de M. Mahieu, INRA).

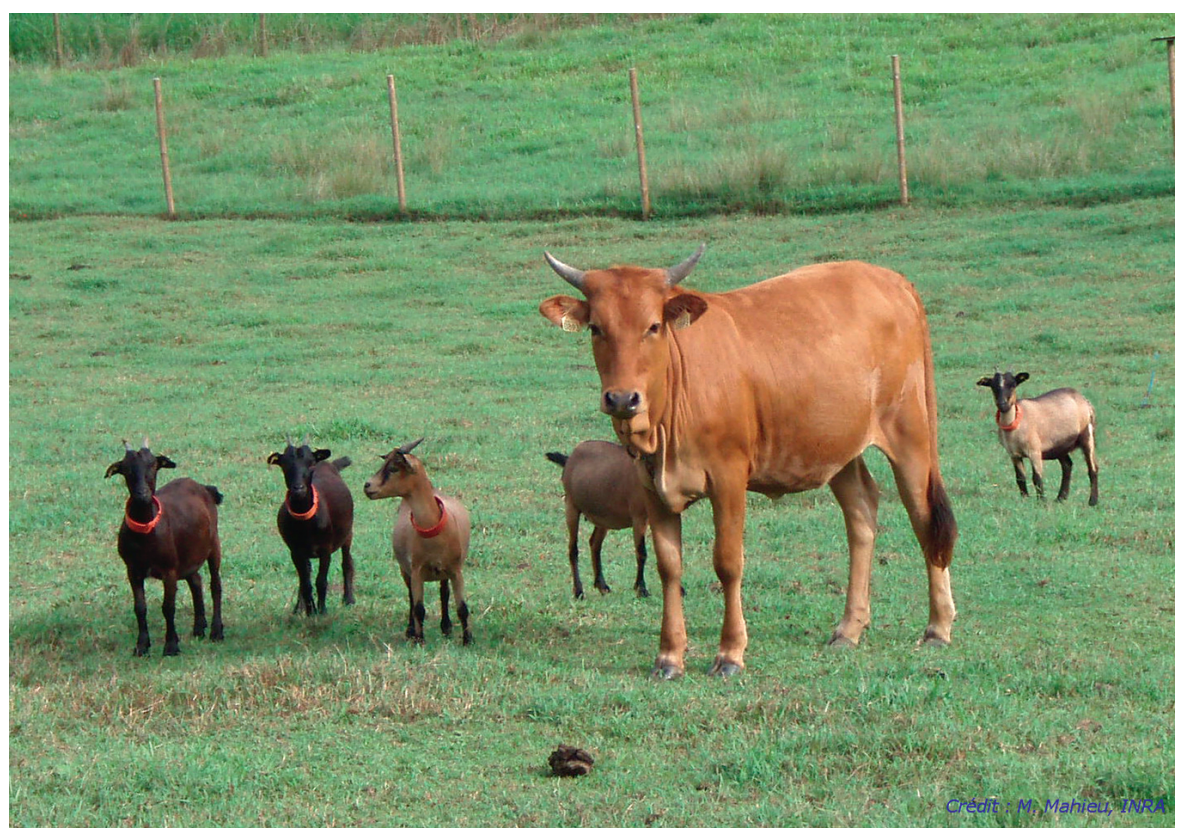

sources végétales natives. Enfin, à plus ou moins long terme, la capacité de résistance de l'hôte peut être stimulée, augmentée par une complémentation alimentaire et la sélection des génotypes les mieux adaptés au risque parasitaire. La résistance génétique aux strongles gastro-intestinaux s'inscrit ainsi dans cette nouvelle démarche de gestion intégrée de la santé et y tient un rôle incontournable en complémentarité avec les stratégies à moins long terme. C'est la voie pour rééquilibrer de façon graduelle les relations hôte-parasite, au profit de l'élevage des petits ruminants.

Cette nouvelle façon d'appréhender la gestion des NGI, et plus généralement 
la santé des animaux, réintroduit plus de complexité dans la conduite d'élevage et considère chaque élevage comme un écosystème particulier. Mahieu (2014) recommande que chaque éleveur puisse, avec un encadrement technique repensé, « choisir parmi la palette d'options possibles dans son contexte épidémiologique, celles qui seront adaptées à sa situation particulière, en considérant les contraintes économiques et organisationnelles aussi bien que les bénéfices en termes de santé animale et de production »".

\section{Conclusion}

Dans cette synthèse, nous avons pu mettre en évidence que la résistance aux NGI pouvait être utilisée en sélection. Cependant l'évaluation de la résistance aux NGI reste une mesure coûteuse, des pistes sont en cours d'exploration pour trouver de bons marqueurs prédictifs (fiables, répétables et bon marché).

Le traitement anthelminthiques est majoritairement utilisé pour lutter contre les infections aux NGI en élevages. Mais aujourd'hui cette stratégie est remise en cause car les parasites s'y adaptent. La sélection génétique pourrait être vue comme une alternative séduisante cependant le risque d'adaptation des vers aux animaux génétiquement résistants reste faible mais non nul. C'est pourquoi il est plutôt proposé de considérer la sélection génétique des animaux dans un contexte de lutte intégrée incluant des approches de traitements sélectifs, nutritionnelles, et de gestion des pâtures. Cette gestion intégrée permet de mieux respecter l'environnement que le seul traitement systématique de tout le troupeau et est économiquement plus favorable pour l'éleveur de petits ruminants au pâturage. L'éleveur redevient premier décisionnaire au sein de son exploitation en s'affranchissant d'un modèle simplifié universel. Cependant, la stratégie intégrée optimale est plus difficile à identifier pour l'éleveur. La modélisation pourra être une aide précieuse pour conseiller l'éleveur dans ses choix en prenant en compte les principales caractéristiques de son élevage et l'ensemble des stratégies de luttes qu'il pourra déployer. Cependant, pour prédire au mieux l'intérêt pour l'éleveur des animaux résistants aux NGI dans des conditions contraignantes (manque de nourritures, période de gestation...), la compétition entre la résistance aux NGI et les autres fonctions de l'animal devra être explorée dans l'avenir. Ces travaux sont en complète adéquation avec les concepts de l'agro-écologie.

\section{Remerciements}

Les auteurs ont bénéficié de financements INRA dans le cadre du projet d'envergure STReP du Méta-Programme Gestion Intégrée de la Santé des Animaux (GISA), de financements FEDERRégion Guadeloupe dans le cadre du projet Agroecotrop (2007-2013), de financement FGE dans le cadre de l'action innovante FENOPAR et OSIRIS et du financement du projet GEMANEMA de l'Institut Carnot Santé animale.

\section{Références}

Assenza F., Elsen JM., Legarra A., Carré C., Sallé G., Robert-Granié C., Moreno C., 2014 Genetic parameters for growth and faecal worm egg count following Haemonchus contortus experimental infestations using pedigree and molecular information. Genet. Select. Evol., 46,13.

Astruc J.M., Fidelle F., Aguerre S., Moreno C., Jacquiet P., 2016. Phenotyping and selecting for genetic resistance to gastro-intestinal parasites in sheep: the case of the Manech French dairy sheep breed. $40^{\text {th }}$ ICAR, Puerto Varas, Chile.

Bambou J.C., de la Chevrotière C., Gunia M., Alexandre G., Arquet R., Mandonnet N., 2010. Genetic correlation between resistance to strongyle natural mixed infection and resistance to Haemonchus contortus experimental infection in Creole goats. 10 $0^{\text {th }}$ Int. Conf. Goats, Recife, Brazil.

Bambou J.C., Larcher T., Cei W., Dumoulin P.J., Mandonnet N., 2013. Effect of experimental infection with haemonchus contortus on parasitological and local cellular responses in resistant and susceptible young creole goats. Biomed. Res. Int., Article ID 902759, 9p.

Bishop S.C., Morris C.A., 2007. Genetics of disease resistance in sheep and goats. Small Rum. Res., 70, 48-59.

Blaes J.L., Mandonnet N., Arquet R., Mahieu M., 2010. A long term experiment of integrated control of nematode parasitism in Creole goats. Adv. Anim. Biosci., 1, 2, 413-414.

Chylinski C., 2014. What makes a gastrointestinal nematode successfull in their sheep host? Exploring the role of the nematode, the sheep host and the farmer. Thèse Sciences de la vie et de la santé, Tours, France, 377p.
Chylinski C., Cortet J., Neveu C., Cabaret J., 2015. Exploring the limitations of pathophysiological indicators used for targeted selective treatment in sheep experimentally infected with Haemonchus contortus. Vet. Parasitol., 207, 85-93.

Ciappesoni G., Gimeno D., Ravagnolo O., 2010 Genetic relationships between faecal worm egg count and production traits in Merino sheep of Uruguay. In: Proc. 9th World Congr. Genet. Appl. Livest. Prod., Leipzig, Germany.

Davies G., Stear M.J., Benothman M. Abuagob O., Kerr A., Mitchell S., Bishop S.C., 2006. Quantitative trait loci associated with parasitic infection in Scottish blackface sheep. Heredity, 96, 252-258.

de La Chevrotière C., Moreno C., Jaquiet P. Mandonnet N., 2011. La sélection génétique pour la maitrise des strongyloses gastro-intestinales des petits ruminants. INRA Prod. Anim. 24, 221-234.

de la Chevrotière C., Bambou J.C., Jaquiet P. Mandonnet N., 2012. Genetic parameters of $\operatorname{IgA}$ and IgE responses against Haemonchus contortus in Creole goats under natural mixed infection. Vet. Parasitol., 186, 337-343.

Demeler J., Ramünke S., Wolken S., Ianiello D., Rinaldi L., Gahutu J.B., Cringoli G., von Samson-Himmelstjerna G., Krücken J., 2013. Discrimination of gastrointestinal nematode eggs from crude fecal egg preparations by inhibitor-resistant conventional and real-time PCR PloS One 8, 45

Djian-Caporalino C., Palloix A., Fazari A., Marteu, N., Barbary A., Abad P., Sage-Palloix A.M., Mateille T., Risso S., Lanza R., Taussig C., Castagnone-Sereno P., 2014. Pyramiding, alternating or mixing: comparative performances of deployment strategies of nematode resistance genes to promote plant resistance efficiency and durability. BMC Plant Biol., 14, 53.

Doeschl-Wilson A.B., Vagenas D., Kyriazakis I., Bishop S.C., 2008. Exploring the assumptions underlying genetic variation in host nematode resistance. Genet. Select. Evol., 40, 241-264.

Geurden T., Hoste H., Jacquiet P., Traversa D., Sotiraki S., di Regalbono A.F., Tzanidakis N., Kostopoulou D., Gaillac C., Privat S., Giangaspero A., Zanardello C., Noé L., Vanimisetti B., Bartram D., 2014. Anthelmintic resistance and multidrug resistance in sheep gastro-intestinal nematodes in France, Greece and Italy. Vet. Parasitol., 201, 59-66.

Gruner L., Bouix J., Brunel J.C., 2004a. High genetic correlation between resistance to Haemonchus contortus and to Trichostrongylus colubriformis in INRA 401 sheep. Vet Parasitol., 119, 51-58.

Gruner L., Bouix J., Vu Tien Khang J., Mandonnet N., Eychenne F., Cortet J., Sauve C., Limouzin C., 2004b. A short-term divergent selection for resistance to Teladorsagia circumcincta in Romanov sheep using natural or artificial challenge. Genet. Select. Evol., 36, 217-242.

Gunia M., Phocas F., Arquet R., Alexandre G., Mandonnet N., 2011. Genetic parameters for body weight, reproduction, and parasite resistance traits in the Creole goat. J. Anim. Sci., 89, 3443-3451

Gunia M., Mandonnet N., Arquet R., Alexandre G., Gourdine J.L., Navès M., Angeon V., Phocas F., 2013a. Economic values of body weight, reproduction and parasite resistance traits for a Creole goat breeding goal. Animal, 7, 22-33. 
Gunia M., Phocas F., Gourdine J.L., Bijma P., Mandonnet N., 2013b. Simulated selection responses for breeding programs including resistance and resilience to parasites in Creole goats. J. Anim. Sci., 91, 572-581.

Harrison G.B.L., Pulford H.D., Doolin E.E., Pernthaner A., Shoemaker C.B., Hein W.R., 2008. Antibodies to surface epitopes of the carbohydrate larval antigen CarLA are associated with passive protection in strongylid nematode challenge infections. Parasite Immunol., 30, 577-584.

Hillrichs K., Schnieder T., Forbes A.B., Simcock D.C., Pedley K.C., Simpson H.V., 2012. Use of fluorescent lectin binding to distinguish Teladorsagia circumcincta and Haemonchus contortus eggs, third-stage larvae and adult worms. Parasitol. Res., 110, 449. 458 .

Hoste H., Sotiraki S., Landau S.Y., Jackson F., Beveridge I., 2010. Goat-Nematode interactions: think differently. Trends Parasitol., 26, 376-381.

Hunt P.W., Kijas J., Ingham A., 2013. Understanding parasitic infection in sheep to design more efficient animal selection strategies. Vet. J., 197, 143-152.

Jacquiet P., Barillet F., Bouix J., François D., Moreno C., Terefe G., 2009. La résistance génétique des ovins aux strongles gastro-intestinaux. Bull. Acad. Vétérinaire de France, 162, 39-46.

Jacquiet P., Sallé G., Grisez C., Prévot F., Liénard E.,Astruc J.M., Lagrifoul G., François D., Moreno C., 2015. Selection of sheep for resistance to gastro-intestinal nematodes in France: where are we and where are we going? $25^{\text {th }}$ Int. Conf. WAAVP, Liverpool, U.K.

Kaplan R.M., Vidyashankar A.N., 2012. An inconvenient truth: global worming and anthelmintic resistance. Vet. Parasitol. 186, 70-78.

Karlsson L.J.E., Greeff J.C., 2006. Selection response in fecal worm egg counts in the Rylington Merino parasite resistant flock. Aust. J. Exp. Agric., 46, 1-3.

Kemper K.E., Emery D.L., Bishop S.C., Oddy H., Hayes B.J., Dominik S., Henshall J.M., Goddard M.E., 2011. The distribution of SNP marker effects for faecal worm egg count in sheep, and the feasibility of using these markers to predict genetic merit for resistance to worm infections. Genet. Res., 93, 203-219.

Kerboeuf D., Hubert J., Hoste H., 1997. Le diagnostic de laboratoire des strongyloses des ruminants. Le Point vétérinaire, 28, 1871-1878.

Laurenson Y.C.S. M, Kyriazakis I., Bishop S.C., 2012. In silico exploration of the impact of pasture larvae contamination and anthelmintic treatment on genetic parameter estimates for parasite resistance in grazing sheep. J. Anim. Sci., 90, 2167-2180.

Lumaret J.P., Errouissi F., 2002. Use of anthelminthics in herbivores and evaluation of risks for the non-target fauna of pastures. Vet. Res., $33,547-562$.

Mahieu M., 2014. Gestion du parasitisme gastrointestinal des petits ruminants en zone tropicale humide. Thèse de doctorat de l'Université de Lorraine, 177pp.
Mahieu M., Arquet R., Fleury J., Coppry O., Marie-Magdeleine C., Boval M., Archimede H., Alexandre G., Bambou J.C., Mandonnet N., 2009. Contrôle intégré du parasitisme gastrointestinal des petits ruminants au pâturage en zone tropicale humide. Renc. Rech. Rum., 16, 265-268.

Mandonnet N., Bachand A., Mahieu A., Arquet R., Baudron F., Abinne-Molza L., Varo H., Aumont G., 2005. Impact on productivity of periparturient rise in fecal egg counts in Creole goats in the humid tropics. Vet. Parasitol., 134, 249-259.

Mandonnet N., Menendez-Buxadera A., Arquet R., Mahieu M., Bachand M., Aumont G., 2006. Genetic variability in resistance to gastro-intestinal strongyles during early lactation in Creole goats. Anim. Sci., 82, 283-287.

Mandonnet N., Mahieu M., Gunia M., Alexandre G., Bambou J.C., 2014. Genetic Resistance to Parasites in Small Ruminants: from Knowledge to Implementation in the Tropics. In: Proc. 10 $0^{\text {th }}$ World Congr. Genet. App1. Livest. Prod., 1-6. 10 ${ }^{\circ}$ World Congr. Genet. Appl. Livest. Prod., Vancouver, Canada.

McDonald B.A., Linde C., 2002. The population genetics of plant pathogens and breeding strategies for durable resistance. Euphytica 124, 163-180.

McRae K.M., McEwan J.C., Dodds K.G., Gemmell N.J., 2014. Signatures of selection in sheep bred for resistance or susceptibility to gastrointestinal nematodes. BMC Genomics, 15,637 .

Mes T.H.M., Eysker M., Ploeger H.W., 2007. A simple, robust and semi-automated parasite egg isolation protocol. Nat. Protoc., 2, 486-489.

Miller J.E., Horohov D.W., 2006. Immunological aspects of nematode parasite control in sheep. J. Anim. Sci., E124-E132.

Moreno C.R., Aguerre S., Bouvier F., Blanchard A., Koch C., Cortet J., Jacquiet P., Sallé G., 2016. Creation of sheep divergent lines for gastro-intestinal parasitism resistance based on a QTL index. EAAP 2016, 67th Ann. Meet. Eur. Fed. Anim. Sci., Belfast U.K.

Morris C.A., Vlassoff A., Bisset S.A., Baker R.L., West C. J., Hurford A.P., 1997. Responses of Romney sheep to selection for resistance or susceptibility to nematode infection. Anim. Sci., 64, 319-329.

Morris C.A., Bisset S.A., Vlassoff A., Wheeler M., West C.J., Devantier B.P., Mackay A.D., 2010. Selecting for resilience in Romney sheep under nematode parasite challenge, 1994-2007. NZ J. Agricult. Res., 53, 245-261.

Otsen M., Hoekstr, R., Plas M.E., Buntjer J.B., Lenstra J.A., Roos M.H., 2001. Amplified fragment length polymorphism analysis of genetic diversity of Haemonchus contortus during selection for drug resistance. Int. J. Parasitol., 31, 1138-1143.

Pickering N.K., Auvray B., Dodds K.G., McEwan J.C., 2015. Genomic prediction and genome-wide association study for dagginess and host internal parasite resistance in New Zealand sheep. BMC Genomics, 16, 1-11.

Riggio V., Matika O., Pong-Wong R., Stear M.J. Bishop S.C., 2013. Genome-wide association and regional heritability mapping to identify loci underlying variation in nematode resistance and body weight in Scottish Blackface lambs. Heredity, 110, 420-429.

Riggio V., Pong-Wong R., Sallé G., Usai M.G., Casu S., Moreno C.R., Matika O., Bishop S.C., 2014. A joint analysis to identify loci underlying variation in nematode resistance in three European sheep populations. J. Anim. Breed. Genet., 131, 426-436.

Robinson N., Piedrafita D., Snibson K., Harrison P., Meeusen E.N., 2010a. Immune cell kinetics in the ovine abomasal mucosa following hyperimmunization and challenge with Haemonchus contortus. Vet. Res., 41, 37.

Robinson N., Pleasance J., Piedrafita D. Meeusen E.N., 2010b. The kinetics of local cytokine and galectin expression after challenge infection with the gastrointestinal nematode, Haemonchus contortus. Int. J. Parasitol., 41, 487-493.

Roeber F., Larsen J.W.A., Anderson N., Campbell A.J.D., Anderson G.A., Gasser R.B., Jex A.R., 2012. A molecular diagnostic tool to replace larval culture in conventional faecal egg count reduction testing in sheep. PloS One 7.

Saccareau M., Moreno C.R., Kyriazakis I., Faivre R., Bishop, S.C., 2016. Modelling gastrointestinal parasitism infection in a sheep flock over two reproductive seasons: in silico exploration and sensitivity analysis. Parasitology, 143, 1509-1531.

Sallé G., Jacquiet P., Gruner L., Cortet J., Sauvé C., Prévot F., Grisez C., Bergeaud J.P., Schibler L., Tircazes A., François D., Pery C., Bouvier F., Thouly J.C., Brunel J.C., Legarra A., Elsen J.M., Bouix J., Rupp R., Moreno C.R., 2012. A genome scan for QTL affecting resistance to Haemonchus contortus in sheep. J. Anim. Sci., 90, 4690-4705.

Sallé G., Moreno C., Boitard S., Ruesche J., Tircazes-Secula A., Bouvier F., Aletru M., Weisbecker J.L., Prévot F., Bergeaud J.P., Trumel C., Grisez C., Liénard E., Jacquiet P., 2014. Functional investigation of a QTL affecting resistance to Haemonchus contortus in sheep. Vet. Res., 45, 68.

Saulai M., Cabaret J., Hostache G., Mandonnet N., Aumont G., 2001. Life-reait evolution of a parasite strongyle nematode in response to host resistance: an experimental approach using Haemonchus contortus in Blackbelly lambs. Genet., Select. Evol., 33, 25-44.

Schmidt E.E., 1998. La fitness du nématode Teladorsagia circumcincta chez des ovins sensibles et résistants au parasitisme. Master 2 Parasitologie, Créteil, Paris, France.

Shaw R.J., Morris C.A., Wheeler M., Tate M., Sutherland I.A., 2012. Salivary IgA: A suitable measure of immunity to gastrointestinal nematodes in sheep. Vet. Parasitol., 186, 109-117.

Wang Y., Li J.V., Saric J., Keiser J., Wu J., Utzinger J., Holmes E., 2010. Advances in metabolic profiling of experimental nematode and trematode infections. Adv. Parasitol., 73, 373-404

Woolaston R. R., Windon R. G. 2001. Selection of sheep for response to Trichostrongylus colubriformis larvae: genetic parameters. Anim. Sci., 73, 41-48. 


\title{
Résumé
}

La résistance génétique des petits ruminants aux infestations par les nématodes gastro-intestinaux (strongles) peut être sélectionnée puisqu'elle est mesurable indirectement par le comptage d'œufs dans les fèces, héritable et génétiquement peu ou pas corrélée aux autres critères de sélection. La mise en place d'une telle sélection dans les schémas nécessitera la création de plateformes permettant de faire face au nombre de mesures à réaliser et imposera le suivi de l'évolution de la communauté de nématodes afin de prévenir un contournement potentiel de la résistance de l'hôte par les parasites. La meilleure pratique pour éviter ce contournement est de considérer la résistance génétique comme un levier d'action à intégrer à d'autres stratégies (la nutrition, la gestion des prairies et les traitements anthelminthiques).

\begin{abstract}
Genetic resistance to infections by gastrointestinal nematodes in small ruminants: a sustainability issue for grass-based production systems

The resistance of small ruminants to gastro-intestinal nematode infection can be included into a genetic selection program. This resistance can be measured by faecal nematode egg counts. This trait is heritable and only lightly correlated to other selected traits. Faecal egg counts should be measured on a large number of small ruminants in order to be included in selection schemes and this requires the services of a phenotyping platform. The evolution of the nematode community under the selection pressure due to an increase in host resistance should be controlled in order to prevent a putative adaptation of nematodes. In order to avoid adaptation of the parasites, an integrated strategy should be implemented and genetic selection for resistance to nematodes should be associated with the use of anthelmintics, as well as an adequate fulfillment of nutritional needs of small ruminants and adequate management of pastures to reduce infection risk.
\end{abstract}

MORENO-ROMIEUX C., SALLÉ G., JACQUIET P., BLANCHARD A., CHYLINSKI C., CABARET J., FRANCOIS D., SACCAREAU M., ASTRUC J.-M., BAMBOU J.-C., MANDONNET N., 2017. La résistance génétique aux infections par les nématodes gastro-intestinaux chez les petits ruminants : un enjeu de durabilité pour les productions à l'herbe. INRA Prod. Anim., 30, 47-56. 